\title{
SEPSISCARE: AVALIAÇÃO DE APLICATIVO MÓVEL NO CUIDADO DE ENFERMAGEM AO PACIENTE COM SEPSE*
}

\author{
Geyza Regina Domingos Melloํㅗ Alacoque Lorenzini Erdmann², Aline Lima Pestana Magalhães³
}

\begin{abstract}
RESUMO: Objetivo: Avaliar um aplicativo móvel para a prevenção, identificação e cuidados de enfermagem ao paciente séptico. Método: Estudo descritivo que utilizou o Processo de Desenvolvimento de Produtos como método para desenvolvimento do aplicativo. Participaram do estudo seis enfermeiras da Unidade de Terapia Intensiva de um hospital público de Santa Catarina. A coleta dos dados ocorreu por meio de entrevistas nos meses de setembro de 2016 a janeiro de 2017, e utilizou-se a análise de conteúdo para análise dos dados. Resultado: 0 aplicativo SepsisCare foi desenvolvido na plataforma Mobincube ${ }^{\circledR}$, com 28 telas, que na concepção dos enfermeiros é um recurso importante para a prática do enfermeiro no cuidado ao paciente com sepse. Conclusão: Acredita-se que o desenvolvimento deste aplicativo móvel facilitará a identificação, prevenção e gerência dos cuidados em torno da sepse, e contribuirá para a atualização desses profissionais, possibilitando, assim, um atendimento mais seguro e de qualidade.

DESCRITORES: Cuidados de enfermagem; Sepse; Aplicativos móveis; Unidades de Terapia Intensiva.
\end{abstract}

\section{SEPSISCARE: EVALUATION OF A MOBILE APPLICATION IN NURSING CARE TO PATIENTS WITH SEPSIS}

ABSTRACT: Objective: Evaluate a mobile application for the prevention, identification and nursing care of septic patients. Method: Descriptive study that used the Product Development Process to develop the application. Six nurses from the Intensive Care Unit of a public hos pital in Santa Catarina participated in the study. Data was collected through interviews from September 2016 to January 2017, and content analysis was used to analyze the data. Result: The SepsisCare application was developed at the Mobincube ${ }^{\circledR}$ platform, with 28 screens, which was considered a valuable resource in the care of patients with sepsis by the participants. Conclusion: It is believed that the development of this mobile application will facilitate the identification, prevention and management of sepsis care and will contribute to the updating of these professionals, thus enabling safer and better quality care.

KEYWORDS: Nursing care; Sepsis; Mobile applications; Intensive Care Unit.

\section{SEPSISCARE: EVALUACIÓN DE APLICACIÓN MÓVIL EN EL CUIDADO DE ENFERMERÍA AL PACIENTE CON SEPSIS}

RESUMEN: Objetivo: Evaluar una aplicación móvil para la prevención, identificación y cuidados de enfermería al paciente séptico. Método: Estudio descriptivo que utilizó el Proceso de Desarrollo de Productos como método para desarrollar la aplicación. Participaron del estudio seis enfermeras de la Unidad de Terapia Intensiva de un hospital público de Santa Catarina. Los datos fueron recolectados mediante entrevistas entre septiembre del 2016 y enero del 2017, y se utilizó el análisis de contenido para analizar los datos. Resultado: La aplicación SepsisCare fue desarrollada en la plataforma Mobincube ${ }^{\circledR}$, con 28 pantallas, que según los enfermeros es un recurso importante para la práctica del enfermero en el cuidado al paciente con sepsis. Conclusión: Se cree que el desarrollo de esta aplicación móvil facilitará la identificación, prevención y gestión de los cuidados relacionados a la sepsis, y contribuirá hacia la actualización de esos profesionales, posibilitando así una atención más seguro y de calidad.

DESCRIPTORES: Atención de enfermería; Sepsis; Aplicaciones móviles; Unidades de cuidados intensivos.

\footnotetext{
${ }^{1}$ Enfermeira. Mestre em Gestão do Cuidado em Saúde e Enfermagem. Enfermeira Emergencista do Hospital Governador Celso Ramos. Florianópolis, SC, Brasil.

${ }^{2}$ Enfermeira. Doutora em Filosofia da Enfermagem. Vice Reitora e Docente da Universidade Federal de Santa Catarina.

Florianópolis, SC, Brasil.

${ }^{3}$ Enfermeira. Doutora em Enfermagem. Docente da Universidade Federal de Santa Catarina. Florianópolis, SC, Brasil.
} 


\section{INTRODUÇÃO}

Os avanços da tecnologia na área da saúde, nas últimas décadas, propiciaram melhorias no tratamento de doenças graves. $O$ acesso aos recursos tecnológicos tem facilitado o cuidado dos pacientes críticos nas unidades de terapia intensiva, incentivando os profissionais de saúde na procura pelo conhecimento e aperfeiçoamento da assistência prestada ao paciente. Todavia, a esperança é a de que este aprimoramento resulte em tratamento seguro e assistência de qualidade ao paciente grave ${ }^{(1)}$.

As Unidades de Terapia Intensiva (UTI) foram criadas com a finalidade de tratar o paciente grave, que necessita de cuidados e observação contínua de equipe especializada. São nesses ambientes que estão os pacientes com maior probabilidade de desenvolvimento de infecções e agravamento das doenças, pois, por muitas vezes, são pacientes imunossuprimidos, com idade avançada, doenças crônicas, condições nutricionais desfavoráveis, permanência prolongada em ambiente hospitalar, aliado à necessidade de vários procedimentos invasivos, como ventilação mecânica invasiva, drenos, cateteres e sondas ou incisões cirúrgicas, que contribuem para infecção ${ }^{(2-4)}$.

A sepse é um processo infeccioso causado por bactéria, vírus ou protozoário levando à disfunção orgânica e à morte. Os sinais e sintomas da Síndrome da Resposta Inflamatória Sistêmica (SIRS) são: cefaleia, náuseas e vômitos, sudorese, calafrios, diarreia, taquipneia, taquicardia, hipertermia ou hipotermia, leucocitose ou leucopenia. Estes preditores, somados à infecção, define a sepse, que adicionada à disfunção orgânica define a sepse grave, e quando aliada com a instabilidade cardiovascular, necessitando de vasopressores, define o choque séptico ${ }^{(5-6)}$.

Os dados epidemiológicos da sepse no Brasil mostram que 17\% dos leitos em UTIs são ocupados por pacientes sépticos e revela, ainda, que 600 mil brasileiros desenvolvem sepse anualmente, mostrando que a incidência de mortalidade por SIRS é de $24,2 \%$, por sepse de 33,9\%; por sepse grave, 46,9\%; e de 52,2\%, por choque séptico ${ }^{(4,7-8)}$. A incidência dessa condição patogênica é estimada em 300 casos por 100.000 pessoas, com elevação de 13\% por ano. A gravidade dessa doença pode ser evidenciada pela alta taxa de mortalidade dos brasileiros (20-50\%) e pela incidência de 30 casos por mil pacientes/dia ${ }^{(9)}$.

Mundialmente, cerca de 1.000 pessoas morrem de sepse a cada hora. Estudo realizado nos Estados Unidos aponta que em 2003 havia 415 mil casos e em 2007 eram 700 mil casos, aumentando, significantemente, o índice de sepse no mundo(10).

Sendo assim, entende-se que a sepse é reconhecidamente um problema de saúde pública que pode ser evitada, caso a equipe de saúde tenha conhecimento suficiente acerca dos sinais e sintomas do início da sepse e cuidados especializados de enfermagem ao paciente, a fim de evitar o seu desenvolvimento, em especial através de tecnologias móveis. Nesse contexto, a tecnologia em saúde pode favorecer a qualidade do cuidado e dos procedimentos realizados ao paciente crítico, tornandoos mais eficazes e precisos, promovendo, desta maneira, a redução dos erros humanos ${ }^{(11)}$.

O uso das ferramentas computacionais foi introduzido na área de enfermagem há mais de 40 anos e continua em crescente expansão, visto que este tipo de auxílio tem sido utilizado para facilitar as tomadas de decisões e trazer agilidade em seus trabalhos. Com o uso das tecnologias móveis observase melhor desempenho profissional, otimização do cuidado, ajudando, assim, na pronta identificação, diagnóstico e tratamento das doenças. Essa verdadeira revolução gera demanda por um novo profissional da enfermagem, não só capaz de utilizar estas novas ferramentas, mas apto para criar e modelar novos instrumentos de cuidado ${ }^{(12-13)}$.

Acredita-se, ainda, que o desenvolvimento de um aplicativo móvel para auxiliar o enfermeiro e sua equipe na identificação precoce da sepse, prevenção e estabelecimento rápido dos cuidados contribuirá para a atualização deste agravo, oferecendo aprendizado aos profissionais de enfermagem, quanto ao atendimento do paciente com quadro séptico instalado, nesse ambiente de alta densidade tecnológica e de cuidados complexos.

Deste modo, o objetivo deste estudo é avaliar um aplicativo móvel (App) para a prevenção, a identificação precoce e o planejamento dos cuidados de enfermagem ao paciente crítico com sepse. 
Trata-se de um estudo descritivo e de produção tecnológica, que utilizou como método o Processo de Desenvolvimento de Produtos (PDP) ${ }^{(14)}$. Este método é formado por fases, tarefas e atividades organizadas, com o objetivo de planejar, desenvolver e construir um novo produto ou a melhoria de um já existente.

O PDP é o modo como as atividades e tarefas progridem para o desenvolvimento dos produtos. Este processo está relacionado com o gerenciamento do conjunto de atividades para desenvolver um produto. Começa com a macrofase de planejamento, na qual são definidos os objetivos a serem alcançados, são definidas as vantagens e desvantagens, e a funcionalidade e viabilidade do produto a ser desenvolvido. Quando esta for concluída, dá-se início a macrofase de desenvolvimento, na qual há detalhes e atividades de desenvolvimento do produto e dimensionamento. Antes que esta macrofase chegue ao fim, é necessário fazer o último teste e finalizar a produção antes do lançamento do produto. Com isso, dá-se o início da macrofase de lançamento do produto, na qual é feito o marketing, a distribuição/publicação e o suporte ao produto, para, desse modo, dar início às atividades propostas $^{(15-16)}$.

O estudo foi realizado em UTI de um hospital público, vinculado à Secretaria de Saúde do Estado de Santa Catarina, na cidade de Florianópolis, que presta ações e serviços de saúde aos indivíduos de nível social diversificado, proveniente de todo o Estado. Possui 14 leitos e atende todas as patologias; porém, as mais frequentes na UTI são: Politraumas, Traumatismo Crânio Encefálico (TCE) e Acidente Vascular Encefálico (AVE). A escolha da UTI se deu por ser o local do hospital com maior número de casos de sepse.

Os participantes do estudo foram os enfermeiros atuantes na UTI do referido hospital, e, como critérios de inclusão, deveriam estar trabalhando no período da coleta dos dados. Como critérios de exclusão, foram considerados os profissionais que estavam afastados de suas atividades na unidade por férias, licenças ou atestados.

Foram realizados a abordagem e o convite individual aos profissionais em seu ambiente de trabalho para participarem do estudo. Dos onze enfermeiros que atuavam na UTI neste período, cinco não participaram, sendo que um estava de férias, um optou por não participar e três não responderam o instrumento de coleta de dados. Sendo assim, totalizou seis participantes.

O aplicativo móvel foi desenvolvido em plataforma on-line e gratuita, disponível para IOS e Android, denominada Mobincube ${ }^{\circledR}$. Foi intitulado pelas pesquisadoras como SepsisCare. A organização do protótipo do aplicativo se deu conforme as fases do PDP que estão descritas abaixo:

Fase 1 - Análise: realizou-se levantamento na literatura acerca do tema "Sepse";

Fase 2 - Design: foi realizada a estruturação do conteúdo e a interface do App, a partir da plataforma Mobincube ${ }^{\circledR}$;

Fase 3 - Desenvolvimento: ocorreu a produção do protótipo do App propriamente dita.

Fase 4 - Implementação: foi impresso o fluxograma do aplicativo desenvolvido e disponibilização aos enfermeiros, para que os mesmos pudessem fazer o manuseio e utilização num período de 15 dias.

Após o desenvolvimento do aplicativo, elaborou-se um questionário para que os enfermeiros avaliassem o conteúdo. Foi realizada a apresentação e o manuseio do aplicativo móvel, a entrega do fluxograma impresso com o conteúdo, e a aplicação do questionário para avaliação. Esse contou com os seguintes questionamentos: 1) Qual sua percepção do protótipo do aplicativo impresso?; 2) A linguagem é adequada?; 3) As tabelas ajudaram na sua compreensão dos tópicos?; 4) Faltou alguma informação sobre a sepse neste protótipo do aplicativo?; 5) Você teve alguma dificuldade na busca/ consulta das informações?; 6) Você tem alguma sugestão que possa ajudar no melhoramento do aplicativo?

Após a devolução do questionário, pelos participantes, foram analisadas as considerações sobre a utilização e o manuseio do fluxograma do aplicativo de dispositivo móvel, e realizadas adequações solicitadas. Após as melhorias e os ajustes necessários, o aplicativo foi novamente disponibilizado para as enfermeiras participantes, para que pudessem reavaliá-lo.

A coleta e análise dos dados foram realizadas entre os meses de setembro de 2016 e janeiro de 2017, e para a análise dos dados utilizou-se a análise de conteúdo(17). 
O estudo foi aprovado pelo Comitê de Ética em Pesquisa com Seres Humanos da Universidade Federal de Santa Catarina (UFSC) e do hospital em que foi realizado o estudo, sob o parecer no 1.539 .079 de 11 de maio de 2016. Os participantes do estudo assinaram o Termo de Consentimento Livre e Esclarecido em duas vias. Para garantir o anonimato, cada participante teve seu nome substituído pela letra $E$, seguido por um número correspondente à ordem de recebimento do instrumento de coleta de dados.

\section{RESUltados}

A amostra foi composta por seis enfermeiras, atuantes em UTI de um hospital público de grande porte do Sul do país. A idade dos participantes estava na faixa etária de 30 a 40 anos, sendo todas do sexo feminino, com tempo de formação e atuação em UTI entre 5 e 10 anos.

O protótipo do aplicativo móvel englobou os seguintes itens: Introdução e Estatísticas da Sepse; Conceito de Sepse; Fatores de Riscos e Alterações Clínicas; Intervenções de Enfermagem; Bundles: Pacote de 3h e Pacote de 6h; Campanha "Sobrevivendo à Sepse"; Vídeos e Referências.

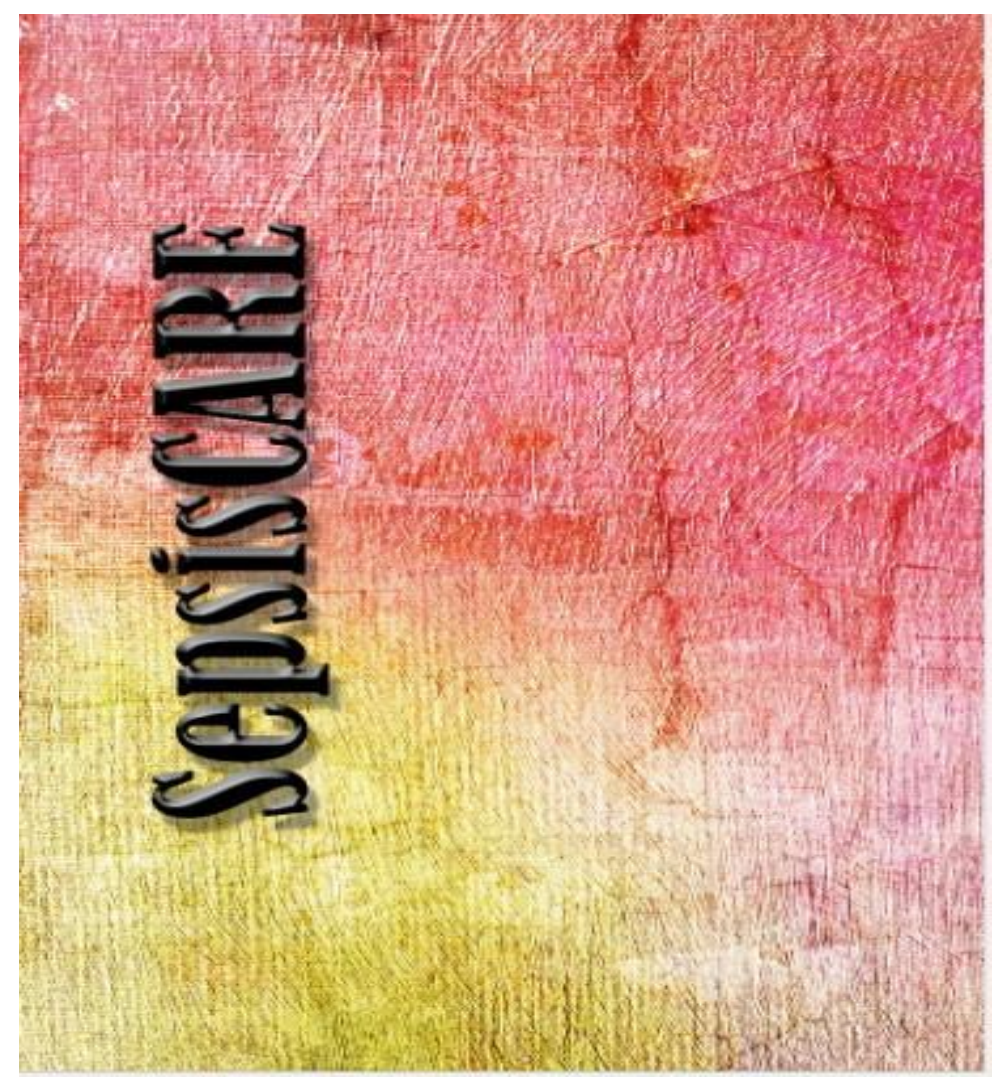

Figura 1 - Capa do aplicativo. Florianópolis, Santa Catarina, Brasil, 2017 


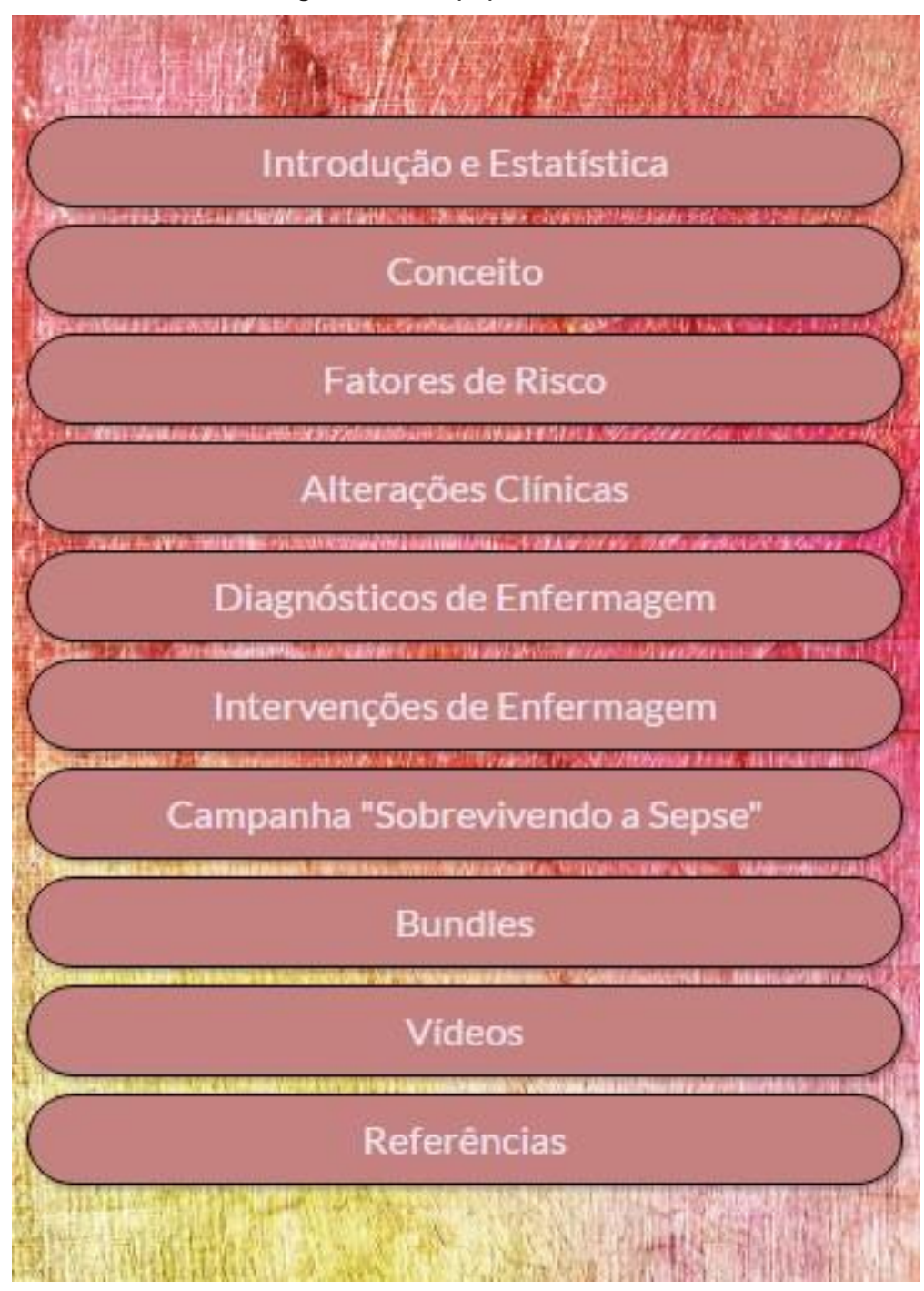

Figura 2 - Menu principal. Florianópolis, Santa Catarina, Brasil, 2017

A seguir, apresentam-se as duas categorias elaboradas: Avaliação do conteúdo do aplicativo e Aprimoramento do conteúdo do aplicativo.

Avaliação do conteúdo do aplicativo

Em relação ao protótipo, todas as participantes salientaram que é um aplicativo interessante e excelente recurso para consulta rápida, além de conter informações importantes para a prática do enfermeiro.

O aplicativo é interessante para consulta. (E2)

Excelente, fácil e rápido acesso. Contém informações importantes para a prática. (E3)

Contém informações e noções resumidas sobre a sepse. (E4)

Quanto à linguagem utilizada, às tabelas e figuras disponibilizadas no aplicativo, todas as participantes referiram que estavam adequadas e auxiliaram na compreensão dos tópicos.

Quando questionadas sobre se sentiram falta de alguma informação sobre a sepse ou sentiram alguma dificuldade em consultar ou buscar informações no protótipo, todas as enfermeiras mencionaram que o protótipo estava adequado e é fácil de manusear. 
Aprimoramento do conteúdo do aplicativo

Como sugestão para o aprimoramento do aplicativo, as enfermeiras destacaram a importância de acrescentar os diagnósticos e a prescrição de enfermagem, pautados, principalmente, na Classificação Internacional para a Prática de Enfermeiros (CIPE ), uma vez que é a terminologia utilizada no cenário do estudo.

Elencar os diagnósticos de Enfermagem aos cuidados. (E1, E2 e E6)

Acrescentar os diagnósticos e prescrição de enfermagem de acordo com a CIPE ${ }^{\circledR}$. (E3)

Elencar os diagnósticos de Enfermagem e associá-los com os cuidados. (E4)

Após a etapa de avaliação do aplicativo, foram realizadas algumas melhorias, destacando-se: Troca de alguns segmentos de texto por figuras, imagens e gráficos, facilitando a visualização e o entendimento rápido do conteúdo; Melhoramento no design do aplicativo; Inclusão dos diagnósticos de enfermagem CIPE ${ }^{\circledR}$ e NANDA ${ }^{\circledR}$ (North American Nursing Diagnosis Association) com menu, possibilitando a alternância entre eles, de modo que outros contextos de trabalho dos enfermeiros, inclusive em outras unidades hospitalares, pudessem fazer uso do aplicativo; Adição de menus secundários individuais (alterações clínicas, diagnósticos de enfermagem e vídeos), nos quais o usuário pode selecionar, dentro do menu de alterações clínicas, aquelas que lhe interessar naquele momento, ou, então, ao entrar no menu de vídeos, clicar naquele de interesse e o mesmo abrir em um programa específico de vídeos; Acesso aos artigos, periódicos e estudos usados no aplicativo no menu principal (Figura 3 e 4).

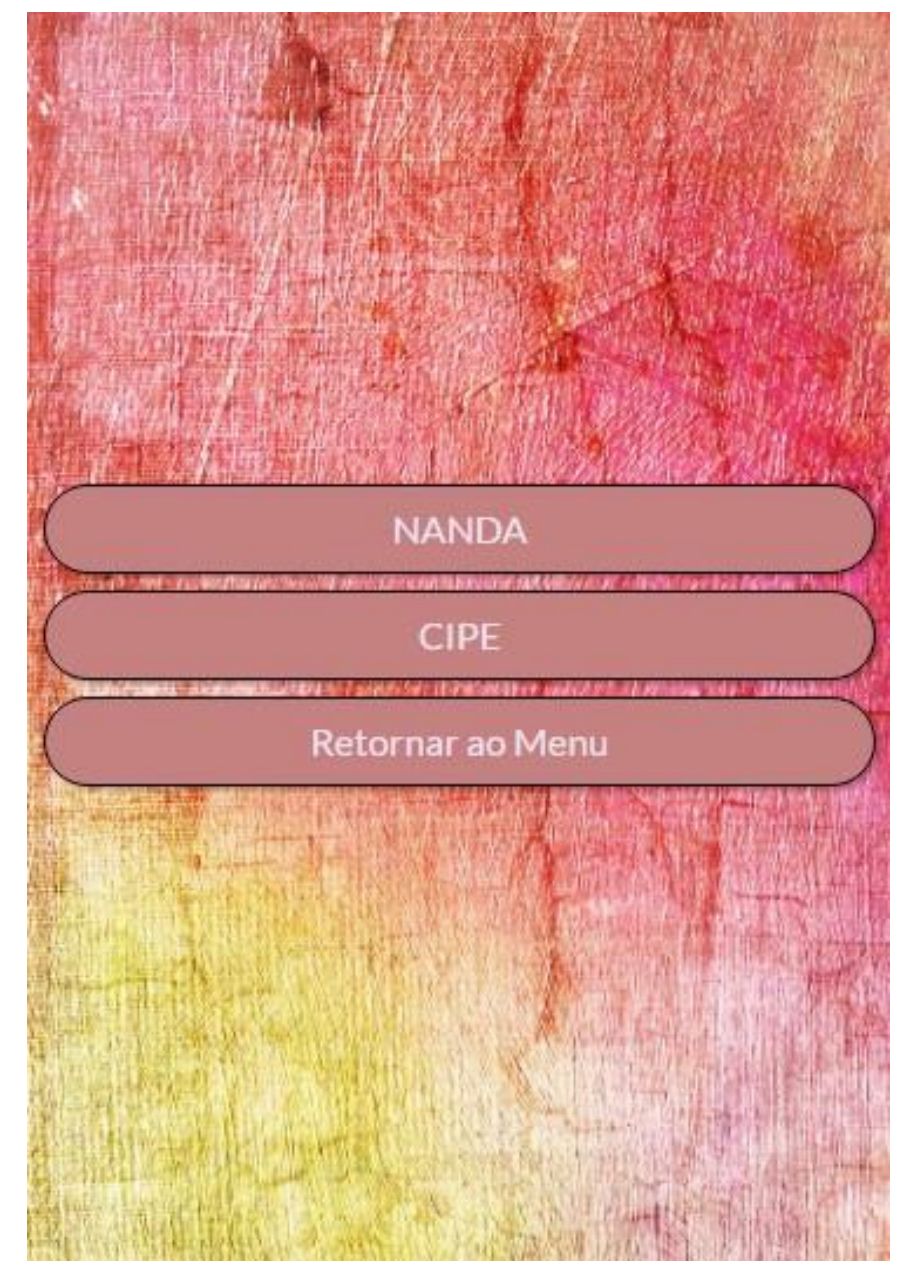

Figura 3 - Menu dos diagnósticos de enfermagem. Florianópolis, Santa Catarina, Brasil, 2017 


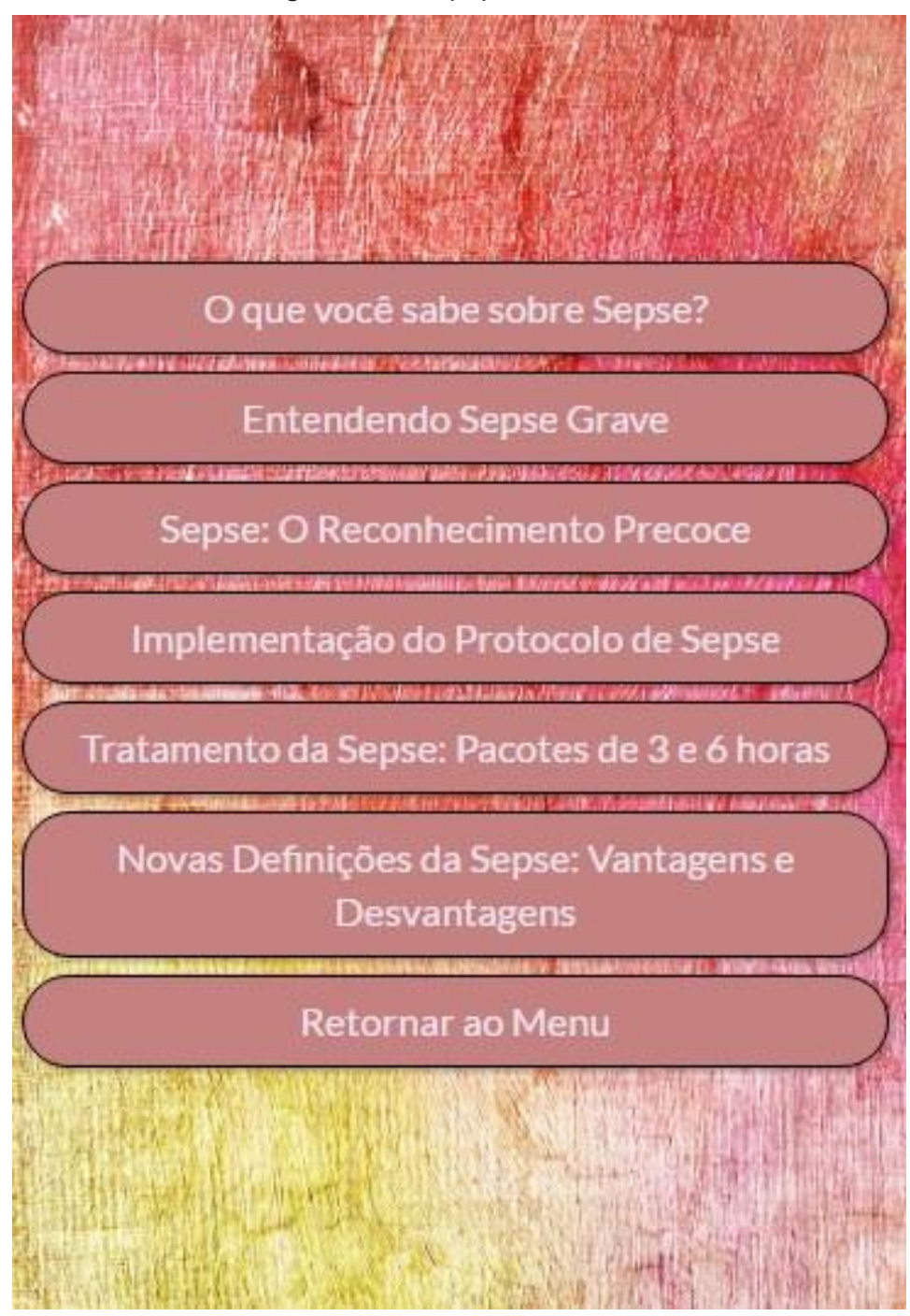

Figura 4 - Menu dos vídeos. Florianópolis, Santa Catarina, Brasil, 2017

Após os aprimoramentos, o aplicativo foi novamente disponibilizado para as seis enfermeiras, para que pudessem reavaliá-lo. Alguns destes relatos estão descritos abaixo:

Ficou mais interessante e útil. As fotos e multimídias acrescentadas, com certeza, ajudaram na compreensão. (E1)

O aplicativo ficou excelente, proporcionando fácil acesso e pesquisas rápidas, além de oferecer opções como vídeo para acrescentar e aprimorar o conhecimento. Achei excelentes as figuras para melhor fixação do conteúdo e os vídeos para mais informações. Os tópicos, vídeos e link das referências, que leva ao artigo utilizado, facilitaram a busca de informação. (E3)

Ele ficou ótimo, eficiente e interessante. A CIPE e NANDAं, os vídeos e as referências, que levam aos artigos facilitaram a busca das informações. (E5, E2)

\section{DISCUSSÃO}

Com relação às características dos participantes, verificou-se que estes achados são condizentes com dados de outros estudos, que caracterizam o perfil do enfermeiro na UTI, em que a população é eminentemente feminina, adultos jovens ${ }^{(18)}$, com formação e tempo de serviço entre 5 e 10 anos $^{(19)}$. 
As tecnologias de informação são diuturnamente mais prevalentes nos hospitais de todo o mundo e, em muitas situações, já consideradas indispensáveis para o cuidado de saúde de qualidade. Nesse contexto, os aplicativos móveis oferecem vantagens, seja na liberdade de movimento e portabilidade que conferem, quanto no nível de investimento mais racional que ensejam. Estudos nacionais e internacionais, consistentemente, sugerem que os aplicativos móveis serão cada vez mais utilizados por profissionais de saúde, para tomadas de decisões ágeis e de melhor qualidade, no cuidado prestado nos ambientes clínicos ${ }^{(20-22)}$.

O aplicativo foi projetado priorizando funcionalidade, mas dando a devida relevância a apresentação gráfica, objetivando simplicidade estética sem perda de conteúdo técnico. A navegação do aplicativo acontece por menus que organizam o conteúdo de forma didática e de fácil acesso, como salientado em estudo recente ${ }^{(12)}$.

Após o período de avaliação do protótipo pelos enfermeiros participantes, observou-se possibilidade de melhorias no fluxo e conteúdo do aplicativo, a fim de torná-lo mais intuitivo. $\mathrm{O}$ uso do aplicativo em ambiente real trouxe informações não previstas na idealização teórica do mesmo. Utilizando estas sugestões e críticas dos participantes, foi possível realizar modificações e inclusão de conteúdos melhor aplicáveis a situações de uso real. Mesmo antes das modificações, os participantes ficaram satisfeitos com o desenho do aplicativo. Destacadamente, foi incluído um novo item referente aos diagnósticos de enfermagem (NANDA ${ }^{\circ}$ e CIPE ${ }^{\circ}$ ), não previstos no modelo original e utilizados diariamente por enfermeiros em UTI.

Pela liberdade conferida por sua mobilidade, o aplicativo pode ser utilizado à beira do leito, permitindo facilmente a classificação do paciente, quanto aos seus parâmetros vitais, e sanando dúvidas em tempo real durante a avaliação do paciente. Esta avaliação em tempo real permite uniformizar e aperfeiçoar a comunicação entre os profissionais envolvidos no cuidado do paciente, proporcionando agilidade na tomada de decisões assistenciais ${ }^{(20)}$.

Em relação à interface de comunicação entre o aplicativo e o usuário, por se tratar de um aplicativo destinado ao uso em situações reais de assistência à saúde, o objetivo é clareza na transmissão das informações. Desta forma, a linguagem utilizada almeja simplicidade e objetividade, evitando demasiado rebuscamento e excesso de informações de cunho não prático, ainda que permita o aprofundamento sobre os tópicos, por meio de links direcionados a artigos científicos, e repositórios de conhecimentos disponíveis livremente na internet.

As informações mais importantes são sumarizadas de forma ilustrativa com recursos multimídia, incluindo figuras, gráficos, tabelas e vídeos. Com auxílio destes recursos didáticos, o entendimento de conceitos complexos torna-se mais simples em curto período de tempo.

Nas seções do aplicativo há maior número de informações enumeradas, como nos Diagnósticos de Enfermagem, em que as mesmas são dispostas em ordem alfabética, facilitando a busca por itens individuais.

$\mathrm{Na}$ análise dos problemas de saúde, encontrados nos pacientes críticos, os diagnósticos de enfermagem são ferramentas essenciais para nortear o julgamento clínico do enfermeiro, possibilitando a pronta elaboração de um plano de cuidado integral ${ }^{(23)}$. Dessa forma, a identificação correta dos Diagnósticos de Enfermagem prevalentes, indubitavelmente permitem intervenções de enfermagem otimizadas para alcançar os resultados esperados ${ }^{(24)}$. No caso dos pacientes com diagnóstico de sepse, estudos mostram que a identificação precoce, associada à tomada de decisão precisa e aos cuidados adequados, pode trazer prognósticos favoráveis ao paciente séptico(4).

Tendo em vista o objetivo de universalidade do uso, o aplicativo foi incrementado com os diagnósticos de enfermagem da NANDA $^{\circ}$ e da CIPE $^{\circ}$. A presença dos dois sistemas de classificação em paralelo permite contemplar não somente o hospital-alvo do estudo, mas diversas instituições e realidades brasileiras.

É importante ressaltar que o uso significativo das tecnologias em saúde tem como objetivos garantir a qualidade e segurança, proporcionando a melhora na comunicação e gerenciamento dos cuidados ${ }^{(25)}$. Esses aplicativos móveis tem sido utilizados nos mais diversos contextos, incluindo universidades para facilitar a compreensão do aluno acerca de algum conteúdo(22), no treinamento de alguma técnica como da ressuscitação cardiopulmonar ${ }^{(26)}$ e também na sociedade, em geral, para auxiliar o autogerenciamento de alguma doença como da asma ${ }^{(27)}$.

Além disso, os atuais ecossistemas de aplicativos móveis permitem a rápida distribuição do aplicativo em diversas plataformas simultaneamente, com baixo custo monetário para o desenvolvedor. Também, permitem sua disponibilização de forma gratuita, facilitando a adesão do mesmo dentro da realidade do nosso Sistema Único de Saúde, sabidamente com financiamento escasso. 


\section{CONCLUSÃO}

Após uma revisão na literatura consistente pela complexidade do tema, compreendeu-se que a sepse continua sendo, no decorrer das últimas décadas, uma síndrome com mortalidade muito elevada, apesar dos avanços obtidos pela medicina no que se refere a seu diagnóstico e sua terapêutica. Logo, o foco de todos os envolvidos, direta ou indiretamente, com o problema deve ser a prevenção e a redução da mortalidade por sepse, através da aquisição de conhecimento quanto ao cuidado prestado.

Neste contexto, o aplicativo desenvolvido e descrito no estudo facilita o reconhecimento ágil do paciente séptico em Unidade de Terapia Intensiva, bem como a adequada qualificação da sua assistência, uniformizando as informações e diminuindo a subjetividade das mesmas.

Desta forma, houve a construção do aplicativo móvel após sugestões das enfermeiras participantes do estudo, como a inclusão dos diagnósticos de enfermagem para o paciente séptico, pois auxiliaria no seu dia a dia na UTI. Baseado na boa receptividade ao aplicativo e nas avaliações positivas quanto a sua funcionalidade, por parte das enfermeiras participantes do estudo, acredita-se que o objetivo principal do estudo foi atingido com sucesso. Analisa-se, contudo, que o aplicativo possui enorme potencial para futuras melhorias e adições de conteúdo.

Reconhece-se que o presente estudo possui limitações, como o pequeno número de enfermeiras que avaliaram o aplicativo piloto e o fato de o teste ter sido realizado em apenas uma instituição. Tais limitações podem ser corrigidas num estudo de maior porte.

No futuro, é possível a incorporação de funcionalidades adicionais ao aplicativo, como escores de risco e de prognóstico para execução rápida à beira do leito. Também, é possível a inclusão de um checklist específico para a sepse, aumentando a segurança do paciente.

Pode-se visualizar, da mesma forma, o desenvolvimento de um módulo de inclusão de informações, para a construção de um banco de dados remoto dos pacientes, facilitando a coleta de informações epidemiológicas sobre sepse, para estudos futuros no tema.

Considera-se que a validação deste aplicativo, em condições reais de uso, frente a métodos tradicionais de avaliação do paciente séptico, é naturalmente a próxima etapa de maturação do mesmo, podendo ser atingida em estudos futuros.

\section{REFERÊNCIAS}

1. Rocha PRS. Fatores associados a mortalidade em pacientes com infecção da corrente sanguínea relacionada a cateter venoso central em uma unidade de terapia intensiva do Distrito Federal [dissertação]. Brasília: Universidade de Brasília; 2012.

2. da Silva PR, Campelo SMA, Sousa LRM, Ferreira AKA, Lima FF, Jacob LMS. Medidas de prevenção da pneumonia associada à ventilação mecânica: uma revisão integrativa. R. Interd. [Internet] 2014;7(2) [acesso em 14 dez 2017]. Disponível: https://revistainterdisciplinar.uninovafapi.edu.br/index.php/revinter/article/view/317/pdf_122.

3. Vieira DF, Kuplich NM, Sottomaior VS, Marona DS. Infecção associada à cateter venoso central. In: Viana RAPP. SEPSE para enfermeiros: as horas de ouro: identificando e cuidando do paciente séptico. 2a ed. São Paulo: Ateneu; 2013. p.177-99.

4. Instituto Latino-Americano para Estudos da Sepse. Conselho Federal de Medicina (CFM). Sepse: um problema de saúde pública. [Internet] Brasília: CFM; 2015 [acesso em 11 nov 2015]. Disponível: http://www.ilas.org.br/ assets/arquivos/upload/Livro-ILAS(Sepse-CFM-ILAS).pdf.

5. Baracho NCV, Lopes GF, Araujo TD, Buissa T, Yano WK. Fatores de risco associados à mortalidade em pacientes com sepse grave e choque séptico na unidade de terapia intensiva de um hospital escola do sul de Minas Gerais. Revista Ciências em Saúde. [Internet] 2011;1(1) [acesso em 11 nov 2015]. Disponível: http://dx.doi.org/10.21876/ rcsfmit.v1i1.17.

6. Oliveira JB, Viana RAPP. Definições e condutas baseadas em evidencia. In: SEPSE para enfermeiros: as horas de ouro: identificando e cuidando do paciente séptico. 2a ed. São Paulo: Ateneu; 2013. p.47-55. 
7. de Carvalho RH, Vieira JF, Gontijo Filho PP, Ribas RM. Sepse, sepse grave e choque séptico: Aspectos clínicos, epidemiológicos e prognóstico em pacientes de Unidade de Terapia Intensiva de um Hospital Universitário. Rev. Soc. Bras. Med. Trop. [Internet] 2010;43(5) [acesso em 11 nov 2015]. Disponível: http://dx.doi.org/10.1590/S003786822010000500025.

8. Reinhart K, Daniels R, Machado FR. O ônus da sepse: uma chamada em apoio ao dia mundial da sepse 2013. Rev. Bras. Ter. Intensiva. [Internet] 2013;25(1) [acesso em 20 nov 2016]. Disponível: http://dx.doi.org/10.1590/ S0103$507 \times 2013000100002$.

9. Rocha LL, Pessoa CMS, Corrêa TD, Pereira AJ, Assunção MSC, Silva E. Conceitos atuais sobre suporte hemodinâmico e terapia em choque séptico. Rev Bras Anestesiol. [Internet] 2015;65(5) [acesso em 27 nov 2017]. Disponível: https://doi.org/10.1016/j.bjan.2015.07.003.

10. Lagu T, Rothberg MB, Shieh MS, Pekow PS, Steingrub JS, Lindenauer PK. Hospitazations, costs and outcomes of severe sepsis in the United States 2003 to 2007. Crit Care Med. [Internet] 2012;40(3) [acesso em 11 nov 2015]. Disponível: http://dx.doi.org/10.1097/CCM.0b013e318232db65.

11. Sousa PAF, Dal Sasso GTM, Barra DCC. Contribuições dos registros eletrônicos para a segurança do paciente em terapia intensiva: uma revisão integrativa. Texto Contexto Enferm. [Internet] 2012;21(4) [acesso em 25 out 2016]. Disponível: http://dx.doi.org/10.1590/S0104-07072012000400030.

12. Grossi LM, Pisa IT, Marin HF. Oncoaudit: desenvolvimento e avaliação de aplicativo para enfermeiros auditores. Acta paul. enferm. [Internet] 2014;27(2) [acesso em ???]. Disponível: http://dx.doi.org/10.1590/19820194201400031.

13. Tibes CMS, Dias JD, Zem-Mascarenhas SH. Aplicativos móveis desenvolvidos para a área da saúde no Brasil: revisão integrativa da literatura. Reme, Rev. Min. Enferm. [Internet] 2014;18(2) [acesso em 13 jan 2017]. Disponível: http://www.dx.doi.org/10.5935/1415-2762.20140035.

14. Salgado EG, Salomon VAP, Mello CHP, Fass FDM, Xavier AF. Modelos de referência para desenvolvimento de produtos: classificação, análise e sugestões para pesquisas futuras. Rev. prod. Online. [Internet] 2010;10(4) [acesso em 09 ago 2016]. Disponível: http://dx.doi.org/10.14488/1676-1901.v10i4.520.

15. Silva GC. Modelo de referência para o processo de desenvolvimento de produto automotivo e diretrizes para seleção de protótipos virtuais e físicos [tese]. São Paulo: Escola Politécnica da Universidade de São Paulo; 2013.

16. Takahashi S, Takahashi VP. Gestão de inovação de produtos: estratégia, processo, organização e conhecimento. Rio de Janeiro: Elsevier; 2007.

17. Bardin L. Análise de conteúdo. 1a edição. São Paulo: Edições 70; 2011.

18. Melech CS, Paganini MC. Avaliação do conhecimento de médico e equipe de enfermagem nas ocorrências de sepse. Rev. Méd. Paraná. [Internet] 2016;3(3) [acesso em 03 fev 2017]. Disponível: http://dx.doi.org/10.5380/ rmu.v3i3.47544.

19. Ramalho Neto JM, Campos DA, Marques LBA, Ramalho CROC, da Nóbrega MML. Concepções de Enfermagem que atuam em unidade de terapia intensiva geral sobre sepse. Cogitare Enferm. [Internet] 2015;20(4) [acesso em 25 jan 2016 ]. Disponível: http://dx.doi.org/10.5380/ce.v20i4.41963.

20. Barra DCC. Processo de enfermagem informatizado em terapia intensiva em ambiente PDA (Personal Digital Assistant) a partir da $\mathrm{CIPE}^{\circledR}$ versão 1.0 [dissertação]. Florianópolis: Universidade Federal de Santa Catarina, Mestrado em Enfermagem, Programa de Pós-Graduação em Enfermagem; 2008.

21. Religioni U, Olejniczak D, Kajak J. Mobile health application as a modern tool of prevention and health education in Poland. Iran J Public Health. [Internet] 2016;45(8) [acesso em 18 jan 2017]. Disponível: https://www. ncbi.nIm.nih.gov/pmc/articles/PMC5139969/.

22. Zhang MW, Tsang T, Cheow E, Ho CSh, Yeong NB, Ho RC. Enabling psychiatrists to be mobile phone app developers: insights into app development methodologies. JMIR Mhealth Uhealth. [Internet] 2014;2(4) [acesso em 14 jan 2017]. Disponível: http://dx.doi.org/10.2196/mhealth.3425. 
23. Okuno MFP, Costa N, Lopes MCBT, Campanharo CRV, Batista REA. Diagnósticos de enfermagem mais utilizados em serviço de emergência. Cogitare Enferm. [Internet] 2015;20(2) [acesso em 14 jan 2017]. Disponível: http://dx.doi.org/10.5380/ce.v20i2.38606.

24. Dutra CSK, Silveira LM, Santos AO, Pereira R, Stabile AM. Diagnóstico de enfermagem prevalentes no paciente internado com sepse no centro de terapia intensiva. Cogitare Enferm. [Internet] 2014;19(4) [acesso em 20 out 2016]. Disponível: http://dx.doi.org/10.5380/ce.v19i4.36801.

25. Madison MP, Staggers N. Electronic health records and the implications for nursing practice. J Nurs Regul. [Internet] 2011;1(4) [acesso em 28 nov 2017]. Disponível: https://doi.org/10.1016/S2155-8256(15)30309-4.

26. Kalz M, Lenssen N, Felzen M, Rossaint R, Tabuenca B, Specht M, et al. Smartphone apps for cardiopulmonary resuscitation training and real incident support: a mixed-methods evaluation study. J Med Internet Res. [Internet] 2014;16(3) [acesso em 20 out 2016]. Disponível: http://dx.doi.org/10.2196/jmir.2951.

27. Miller $\underline{L}$, Schüz $\underline{B}$, Walters $\underline{J}$, Walters $\underline{E} \underline{H}$. Mobile Technology Interventions for Asthma Self-Management: Systematic Review and Meta-Analysis. JMIR Mhealth Uhealth. [Internet] 2017;5(5) [acesso em 07 dez 2017]. Disponível: http://dx.doi.org/10.2196/mhealth.7168. 